\title{
Arterio-enteric Fistula: a case report
}

\author{
${ }^{1}$ Anil Degaonkar, ${ }^{2}$ Nikhil Bhamare, ${ }^{3}$ Mandar Tilak \\ ${ }^{1}$ Assistant Professor, ${ }^{2}$ Resident, ${ }^{3}$ Assist professor\} Dept of Surgery, Dr Shankarrao Chavan Government \\ Medical CollegeNanded (MS) INDIA.
}

\begin{abstract}
Gastro-intestinal (GI) bleeding may originate anywhere from oral cavity to anus from the innocuous one like peptic ulcers to capricious lesion like ca colon. Classically if the cause of bleeding is somewhere below the ligament of Treitz, the stools are maroon or bright red in colour and it is described as lower GI bleeding or hematochezia. Arterioenteric fistula signifies a rare but important cause of massive lower GI bleeding .The vexing problem lies in proper and timely diagnosis of this condition.A keen clinical acumen and proper use of tests lead to accurate diagnosis and prompt treatment and can be lifesaving for the patient by treating significant ongoing bloodloss. We wish to report such a case of an arterio-enteric fistula between artery of broad ligament of uterus and terminal ileum diagnosed and successfully treated at our institute .

Keywords: arterioenteric fistula, massive GI bleed, rare cause,
\end{abstract}

\section{Introduction:}

Arterioenteric fistula is a anomalous communication between artery \& gastrointestinal tract. It is a rare cause of massive lower GI bleeding with the dreaded aortoenteric fistula leading to massive and many times fatal GI bleed.

\section{CASE REPORT}

A 28 yr lady presented with complains of recurrent per rectal bleeding and severe anemia. She had undergone exploration for ectopic pregnancy 1 month back at a private hospital..On $8^{\text {th }}$ post operative day she had complained of three episodes of per rectal bleeding which was associated with giddiness and profound weaknessHer sigmoidoscopy had been done and no abnormality was detected.Patient had been transfused, stabilised and subsequently discharged .She whad been stable for the next 20 days and now presented with above complains of recurrent per rectal bleeding and severe anemia. Patient was investigated.Hematological investigations showed low haemoglobin $\mathrm{hb}-5 \mathrm{gm} \%$ with reticulocytosis. Platelets were adequate and bleeding and clotting time was normal. Serum Beta hCG was elevated .Upper GI scopy showed no abnormality. On colonoscopy only significant finding observed weas presence of blood clots near caecum.USG was suggestive of heterogeneous mass in right adnexa with left ovarian cyst.CT confirmed the ultrasound findings. .Pt was transfused with 3 pints of PCV .Her condition improved and she remained stable for next 8 days .On $9^{\text {th }}$ day she developed three episodes of massive per rectal bleed and went into hypovolemic shock..Her pulse rate was 146/min, BP 90/60 mm of hg.patient was pale and dehydrated.she did not have any hematemesis and ryle's tube aspirate was clear. Due to absence of hematemesis and pain in epigastrium upper GI bleeding was less likely. Also patient had a history of abdominal exploration. Thus a arterioenteric fistula was suspected. Advanced investigation modalities like angiography and technicium99 labelled rbc scan was unavailable at our institute. Hence a decision for emergency exploratory laparotomy after proper resuscitation of the patient was taken.

On exploratory laparotomy. There was evidence of adherent ileum to the posterior aspect of the broad lig. of uterus ( site at which the gestational sac of previous ectopic was present.) Ileum was separated form adhesion site. Erosion of ileum wall with bleeder at site of adhesion to broad ligament was found. The site of adhesion on the broad ligament showed necrosis .

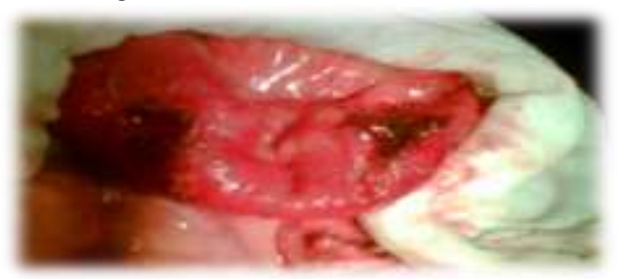

FIG.1 INVOLVED ILEAL SEGMENT

Thus this was a case of arterioenteric fistula between the adherent ileum and a branch of the ovarian artery supplying the broad ligament .All bowell adhesions were separated.The bleeder as ligated hysterectomy was done. Adherent and eroded segment of ileum was resected. Intra-operative enteroscopy both antegrade and retrograde was done in the ileum to rule out any othe site of GI bleed. Ileo-ileal anastomosis was done. 
Postoperatively the patient was monitored in Surgical intensive care unit. Patient received 3 blood transfusions. Patient's general condition improved steadily and patient was discharged on $14^{\text {th }}$ post operative day.

Gross observation of resected specimen of ileum and uterus with broad ligament as well as histopathological analysis suggested a arterio-enteric fistul between the ileum and the broad ligament of the uterus,
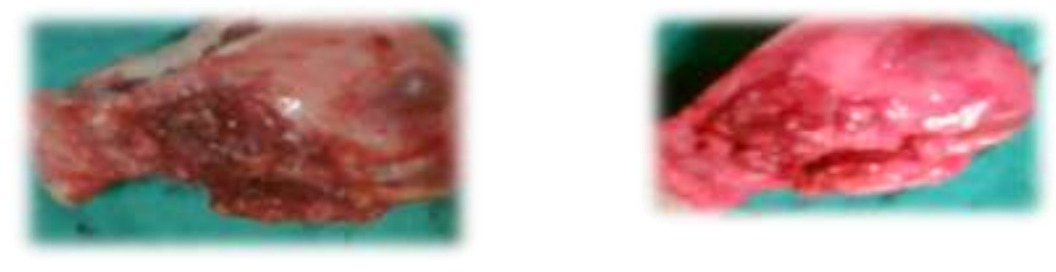

FIG.2. \& 3 RESECTED SPECIMEN OF UTERUS (GROSS)

\section{Discussion}

The causes of lower GI bleeding shows a geographical variation, wit colonic diverticulitis and vascular ectasia of colon are the most common causes in the West [1]. Idiopathic ulcerative colitis, acute colitis, colonic polyps, solitary rectal ulcer, colonic carcinoma, ileal and colonic tuberculosis, non steroidal anti-inflammatory drug enteropathy and enteric fever are the major causes of lower GI bleeding in India [2,3]. Small bowel sources and other colonic pathologies like small bowel diverticular disease, inflammatory bowel diseases, neoplasia of small and large bowel, angiodysplasia, aorto-enteric fistula, ischaemic and radiation colitis are uncommon causes but pose a challenge to the clinician in making correct preoperative diagnosis [3,4].

It is imperative to localize the source of bleeding preoperatively for successful treatment. Only rarely does laparotomy need to be performed in emergency without knowledge of the site of hemorrhage [5]. The diagnostic work-up should be done as soon as the resuscitation is over and the general condition stabilizes. The first step is nasogastric aspiration and upper gastrointestinal endoscopy (esophago-gastroduodenoscopy) to rule out upper GI hemorrhage since peptic ulcer bleeding may be the cause of hematochezia and malena. Proctoscopy and sigmoidoscopy (rigid or flexible) are relatively simple procedures to exclude hemorrhage below the peritoneal reflection such as bleeding internal hemorrhoids, rectal polyps and growths. Colonoscopy, visceral angiography and abdominal scintigraphy with $99 \mathrm{~m}$ Tc labeled RBCs are three useful tests for localization [5].Colonoscopy may be most useful if the bleeding has stopped or at least slowed substantially.

Selective visceral angiography is very useful if the patient is having active bleeding to locate exact site of bleeding.Abdominal scintigraphy with $99 \mathrm{~m}$ Tc labeled RBC infusion is helpful in delineating the site of bleeding when bleeding is intermittent and at a rate below that which is detectable by angiography.

In case the above facilities are not at hand, a combination of sigmoidoscopy and air contrast barium enema may be tried if patient's general condition permits [6]. We have searched the literature on the subject and have come across some pathological conditions leading to fistula formation between aorta and the intestine [7,8]. Abdominal aortic aneurysm and infective aortitis may lead to primary aorto-enteric fistula but in most of the cases the bleeding occurs due to erosion of aortic vascular prosthesis through the wall of distal duodenam due to prolonged contact between prosthetic graft and a fixed segment of intestine (secondary aorto-enteric fistula). Bleeding may occur due to dehiscence of the anastomosis with bleeding into the bowel lumen from the edges of the eroded intestine [7].

The intestine may take blood supply from the anterior abdominal wall due to adhesion formation as seen sometimes as a sequele of mesenteric venous thrombosis, malignancies and fungal infection. A case has been reported where mucomycosal invasion took place into the iliac artery causing severe haemorrhage, in a case of non Hodgkin's lymphoma receiving chemotherapy [8]. In this case, the cause of arterio-enteric fistula formation was due to continued trophoblastic activity of incompletely removed gestational sac \& as the syncytiotrophoblast has invading property[9]. it has eroded the ileum \& fistula is formed between uterine artery of broad ligament $\&$ terminal ileum. Monitoring of trophoblastic activity can be done by estimation of serum beta HCG levels[10].

In the above case report we have presented a case of arterio-enteric fistula between ileum and broad ligament of the uterus. This is a very rare pathology and has seldom been reportedWe hypothesize that the ectopic pregnancy and exploratory laparotomy for the same probably created a inflamed and eroded surface on the broad ligament where ileum adhered. The ileal wall was further eroded .The inflammatory process exposed a artery on the broad ligament and this adhered to the ileum cresting the arterioenteric fistula 


\section{Reference}

[1] DeMarkles MP, Murphy JR. Acute lower gastrointestinal bleeding. Med Clin North Am 1993 Sep; 77(5):1085-100.

[2] Goenka MK, Kochhar R, Mehata SK. Spectrum of lower gastrointestinal hemorrhage: an endoscopic study of 166 patients. Indian J Gastroenterology 1993 Oct; 12(4):129-31.

[3] Anand AC, Patnaik PK, Bhalla VP, Choudhary, et al. Massive lower intestinal bleeding - a decade of experience. Trop Gastroenterol 2001 Jul-Sep;22(3):131-4.

[4] Miller LS, Barbarvech C, Friedman LS. Less frequent causes of lower gastrointestinal bleeding. Gastroenterol Clin North Am 1994 Mar;23(1):21-52.

[5] Gracia Osogobio S, Remes Troche JM, et al. Surgical treatment of lower digestive tract hemorrhage - Experience at the Institute Nacional de ciencias Medicas Y Nutricion Salvador Zubiran.Rev Invest Clin 2002 Mar-Apr; 52(2): 119-24.

[6] Mark HB, Robert B, Mark B. Merk Manual Diagnosis and Therapy. Seventeenth Edition Sec - 3, Ch-22. Gastrointestinal Bleeding.

[7] Kahhlke V, Brossmanm J, Klomp HJ. Lethal hemorrhage caused by aortoenteric fistula following endovascular stent implant. Cardiovasc Intervent. Radiol 2002 May-Jun:25(3):205-7.

[8] Mir N, Edmonson R, Yeghen T, Rashid H. Gastrointestinal mucormycosis complicated by arterio-enteric fistula in a patient with non-Hodgkin's lymphoma. Clin Lab Haematology 2000 Feb;22(1):441-4.

[9] Datta; textbook of gynaecology; $6^{\text {th }}$ edtn;chapter 2;page no.23

[10] Datta; textbook of gynaecology; $6^{\text {th }}$ edtn; chapter 15;page no.186. 